\title{
Local Diagnosis Algorithms for Multiprocessor Systems Under the Comparison Diagnosis Model
}

\author{
Cheng-Kuan Lin, Yuan-Hsiang Teng, Jimmy J. M. Tan, and Lih-Hsing Hsu
}

\begin{abstract}
An efficient diagnosis is very important for a multiprocessor system. The ability to identify all the faulty devices in a multiprocessor system is known as diagnosability. In the comparison model, the diagnosis is performed by sending two identical signals from a processor to a pair of distinct neighbors, and then comparing their responses. Sengupta and Dahbura proposed a polynomial-time algorithm with time complexity $O\left(N^{5}\right)$ to diagnose a system with a total number $N$ of processors under the comparison model. Recently, some concepts, such as the conditional diagnosability and the local diagnosability, are concerned with the measure which is able to better reflect fault patterns in real systems. In this paper, we propose a specific structure, the balanced wind-bell-tree, and give an algorithm to determine the fault status of each processor for conditional local diagnosis under the comparison model. According to our results, a specific $t$-connected network with the balanced wind-bell-tree structure is conditionally $(2 t-1)^{*}$-diagnosable, and the time complexity to diagnose all the faulty processors is $O\left(N(\log N)^{2}\right)$ with our algorithm, where $N$ is the total number of the processors in the network.
\end{abstract}

Index Terms - Comparison diagnosis model, conditional diagnosability, local diagnosis, system diagnosis.

\section{ACRONYMS AND ABBREVIATIONS}

$\begin{array}{ll}\text { NoC } & \text { network-on-chip } \\ \text { PMC } & \text { Preparata, Metze, Chien } \\ \text { MM } & \text { Maeng, Malek } \\ \text { VLSI } & \text { very large scale integration }\end{array}$

NOTATIONS

$G=(V, E) \quad$ a graph, where $V$ is a finite set, and $E$ is a subset of $\{\{u, v\} \mid\{u, v\}$ is an unordered pair of $V$ \}

$N_{G}(u) \quad$ the neighborhood set $\{v \mid\{u, v\} \in E(G)\}$

$\operatorname{deg}_{G}(v) \quad$ the degree of $v$ in $G$

Manuscript received December 04, 2011; revised November 01, 2012; accepted May 21, 2013. Date of publication October 17, 2013; date of current version November 25, 2013. Associate Editor: S. Shieh.

C.-K. Lin is with the Institute of Information Science, Academia Sinica, Taipei City, Taiwan 11529, R.O.C. (e-mail: cklin@iis.sinica.edu.tw).

Y.-H. Teng is with Department of Computer Science and Information Engineering, Hungkuang University, Taichung City, Taiwan 433, R.O.C. (e-mail: yhteng@sunrise.hk.edu.tw).

J. J. M. Tan is with Department of Computer Science, National Chiao Tung University, Hsinchu City, Taiwan 300, R.O.C. (e-mail: jmtan@cs.nctu.edu.tw).

L.-H. Hsu is with Department of Computer Science and Information Engineering, Providence University, Taichung City, Taiwan 433, R.O.C. (e-mail: lhhsu@cs.pu.edu.tw).

Color versions of one or more of the figures in this paper are available online at http://ieeexplore.ieee.org.

Digital Object Identifier 10.1109/TR.2013.2285031
$M=(V, C) \quad$ a multigraph, where $V$ represents the vertex set, and $C$ represents the labeled-edge set

$(u, v)_{w} \quad$ a labeled-edge, which represents that the vertices $u$ and $v$ are compared by $w$

$r_{w}(u, v) \quad$ the result of comparing vertices $u$ and $v$ by $w$

$\tau(G)$

$\tau^{c}(G)$

$\tau_{G}^{*}(v)$

$\tau_{G}^{c}(v)$

$\sigma(F)$

$F_{1} \triangle F_{2}$

the diagnosability of $G$

the conditional diagnosability of $G$

the local diagnosability of $v$ in $G$

the conditional local diagnosability of $v$ in $G$ the set of syndromes which could be generated if $F$ is the set of faulty vertices $\equiv\left(F_{1}-F_{2}\right) \cup\left(F_{2}-F_{1}\right)$, the symmetric difference between $F_{1}$ and $F_{2}$.

$W B_{G}(x ; t) \quad$ a wind-bell-tree of order $t$ rooted at $x$ in $G$

$B W B_{G}(u ; t) \quad$ a balanced wind-bell-tree of order $t$ rooted at $u$ in $G$

$S_{n}$

$\mathbf{u}=$ an $n$-dimensional star graph

$u_{1} u_{2} \ldots u_{n}$

$(\mathbf{u})_{i}$

$(\mathbf{u})^{i}$

$S_{n}^{i: j}$

a vertex in $S_{n}$

the $i$ th component $u_{i}$ of $\mathbf{u}$

the unique $i$-neighbor of $\mathbf{u}$

the subgraph of $S_{n}$ induced by vertices $\mathbf{u}$ with $(\mathbf{u})_{i}=j$

\section{INTRODUCTION}

C LOUD computing and high-speed multiprocessor systems have gained popularity in computer technology. Cloud computing is a computing model which shares the resources. In the multiprocess system, which is a component of a Cloud architecture, sets of processors can operate many programs simultaneously. The reliability of these systems is crucial because even a few malfunctions would disable service for many customers. Whenever devices are found to be faulty, they should be replaced with fault-free ones as soon as possible to guarantee that the system can work properly. Thus the ability of identifying all the faulty devices in a multiprocessor system is very important. This is known as system diagnosis. The diagnosability is the maximum number of faulty devices that can be identified correctly. A system is $t$-diagnosable if at most $t$ faulty processors can be identified precisely. Many results about the system diagnosis and the diagnosability have been proposed in literature [6], [8]-[12], [15], [18]-[22], [24], [32]. 
A multiprocessor system consists of processors and communication links between processors. In practice, most multiprocessor systems are based on an underlying bus structure, or fabric, and are perfectly feasible for a central test controller (a physically independent processor acting as a controller) to check each processor in the system. In such a scheme, the central controller itself can be tested externally. Some research is related to the issue of network-on-chip (NoC); for example, Pande et al. [28] developed an evaluation methodology to compare the performance and characteristics of a variety of NoC topologies; Bartic et al. [3] presented an NoC design which is suitable for building networks with irregular topologies.

Throughout this paper, the underlying topology of a multiprocessor system is modeled as a graph; each processor is represented by a vertex, and the communication bus, or fabric, is represented by a single edge between two vertices. A diagnosis testing signal is supposed to be delivered from one vertex to another through the communication bus. A system performs a so-called system-level diagnosis by making each processor act as a tester to test each of the directly connected ones. It is noticed that such a scheme contains no central test controller. Several well-known approaches to system diagnosis have been developed. Two fundamental approaches are tested-based diagnosis and comparison-based diagnosis. Preparata, Metze, and Chien [29] proposed a model for system diagnosis, called the PMC model. The PMC model is the tested-based diagnosis with a processor performing the diagnosis by testing on the neighboring processors via the links between them. Another classic approach using the comparison-based diagnosis, called the comparison diagnosis model, was first proposed by Maeng and Malek [24], [25], thus termed the Maeng, Malek (MM) model. In the MM model, the diagnosis is performed by sending two identical tasks from a processor to a pair of distinct neighbors, and then comparing their responses. Sengupta and Dahbura [30] gave an $O\left(N^{5}\right)$ diagnosis algorithm to diagnose a system of $N$ processors under the MM model. Several different studies about the MM model also have been proposed in [5], [7], [9], [13], [14], [33].

In some circumstances, we are only concerned about some substructure of a multiprocessor system, which is implementable in very large scale integration (VLSI). Such a substructure, for example, can be a ring, a path, a tree, a mesh, and so on. If all processors in these substructures can be guaranteed to be fault free, a procedure is still workable even though there are many faulty processors in the remaining part of the system. Thus, the local substructure plays a more critical role than the global status of the entire system. Motivated by such a concept, Hsu and Tan [16] presented an elegant measure of diagnosability, known as local diagnosability, to identify the diagnosability of a system by computing the local diagnosability with respect to each individual processor.

In classical measures of system-level diagnosability for multiprocessor systems, it has generally been assumed that any subset of processors can potentially fail at the same time. As a consequence, the diagnosability of a system is upper bounded by its minimum degree. In practice, processors in many systems are connected sparsely. Thus, some research addresses the measures that can better reflect fault patterns in real systems. For instance, Lai et al. proposed conditional diagnosability in [20], which restrains all neighboring processors of a processor from being faulty at the same time. Lin et al. gave a useful structure in [23] to determine its conditional local diagnosability under the PMC model. In this paper, we address conditional local diagnosability under the MM model. We propose a specific structure named the balanced wind-bell-tree, and give an algorithm to efficiently diagnose a vertex in the system. With our algorithm, the faulty or fault-free status of a vertex can be identified correctly if the total number of faulty vertices does not exceed $2 t-1$, where $t$ is the connectivity of the system.

The rest of this paper is organized as follows. Section II provides preliminary background for system diagnosis and graph-theoretic terminology. In Section III, we propose a specific structure for local diagnosis, and present a local diagnosis algorithm under the MM model. In Section IV, we give the definition of the balanced wind-bell-tree, and propose an algorithm for conditional local diagnosis under the MM model. With our results, we give an application in Section V. In the final section, we present our conclusions, and measure the time complexity of our proposed algorithm.

\section{PReliminaries}

The underlying topology of a multiprocessor system is usually modeled as a graph, whose vertex set, and edge set represent the set of all processors, and the set of all communication links between processors, respectively. For the graph definitions and notation, we follow [17] and the Notation section. Two vertices $u$ and $v$ in a graph $G=(V, E)$ are adjacent if $\{u, v\} \in E$; we say $u$ is a neighbor of $v$, and vice versa. The degree of a vertex $v$ in a graph $G$ is the number of edges incident to $v$.

\section{A. System Diagnosis}

The MM model is proposed by Maeng and Malek in [24], [25]. Under the MM model, the system diagnosis is performed by a specific testing procedure. For each processor $w$, which has two distinct links to two other processors $u$ and $v$, the diagnosis can be performed by simultaneously sending two identical signals from $w$ to $u$ and from $w$ to $v$, and then comparing their returning responses in the reverse direction. The comparison scheme of the system can be modeled as a multigraph $M=$ $(V, C)$. For $(u, v)_{w} \in C$, if the outputs of $u$ and $v$ agree, we have $r_{w}(u, v)=0$; otherwise, $r_{w}(u, v)=1$. If $r_{w}(u, v)=0$, and $w$ is fault-free, then both $u$ and $v$ are fault-free. If $r_{w}(u, v)=1$, then at least one of $u, v$, and $w$ must be faulty. If $w$ is faulty, then the result of the comparison is unreliable, and the exact status of $u$ and $v$ are unknown. The complete result of all comparisons, defined as a function $\sigma: C \rightarrow\{0,1\}$, is called the syndrome of the diagnosis. The set $F \subseteq V$ of all faulty processors in a graph $G=(V, E)$ is called a faulty set. Two distinct faulty sets $F_{1}$ and $F_{2}$ of $V$ are said to be distinguishable if $\sigma\left(F_{1}\right) \cap \sigma\left(F_{2}\right)=\emptyset$; otherwise, $F_{1}$ and $F_{2}$ are said to be indistinguishable. Clearly, a system is $t$-diagnosable iff each pair of sets $F_{1}$ and $F_{2}$ are distinguishable with $\left|F_{1}\right| \leq t$ and $\left|F_{2}\right| \leq t$. There are several different ways to verify a system to be $t$-diagnosable under the comparison approach. The following theorem given by Sengupta and Dahbura [30] is a necessary and sufficient condition for ensuring distinguishability. 


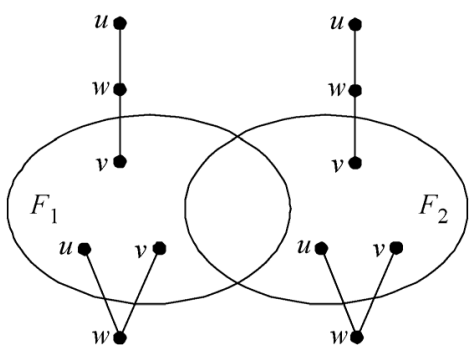

Fig. 1. An illustration for Theorem 1.

Theorem 1: [30] For any $F_{1}, F_{2} \subset V$ and $F_{1} \neq F_{2},\left(F_{1}, F_{2}\right)$ is a distinguishable pair iff at least one of the following conditions is satisfied.

1) $\exists u, w \in V-\left(F_{1} \cup F_{2}\right)$ and $\exists v \in F_{1} \triangle F_{2}$ such that $(u, v)_{w} \in C$.

2) $\exists u, v \in F_{1}-F_{2}$ and $\exists w \in V-\left(F_{1} \cup F_{2}\right)$ such that $(u, v)_{w} \in C$.

3) $\exists u, v \in F_{2}-F_{1}$ and $\exists w \in V-\left(F_{1} \cup F_{2}\right)$ such that $(u, v)_{w} \in C$.

See Fig. 1 for an illustration of the theorem.

For a vertex set $U \subset V(G)$, we define $T(G, U)$ to be the set $\left\{v \mid(u, v)_{w} \in C,\{u, w\} \subseteq U\right.$ and $\left.v \in V(G)-U\right\}$. In [30], Sengupta and Dahbura also proposed a sufficient condition for a system being $t$-diagnosable.

Theorem 2: [30] A system $G=(V, E)$ with $N$ vertices is $t$-diagnosable if

1) $N \geq 2 t+1$

2) each vertex has order at least $t$, and

3) for each $U \subset V(G)$ such that $|U|=N-2 t+p$ and $0 \leq p \leq t-1,|T(G, U)|>p$.

\section{B. Local Diagnosis}

The probabilities of processor failures in a multiprocessor system are identical and statically independent under the random-fault model. Let $v \in V(G)$. It is intuitive to observe that $\left(N_{G}(v),\{v\} \cup N_{G}(v)\right)$ forms an indistinguishable pair of faulty sets. That is, the conventional diagnosability that has been addressed by many researchers mainly describes the global status of a system under the random-fault model. Instead, Hsu and Tan [16] presented the concept of local diagnosability. The research about local diagnosability concerns with the local connective substructure in a system. That is, for a processor $v$ in a system, it is only required to determine whether $v$ is faulty or not. Given a syndrome $\sigma(F)$ produced by a faulty set $F$ with $v \in F$ and $|F| \leq t$, a graph $G$ is $t^{*}$-diagnosable at vertex $v$ if every faulty set $F^{\prime}$ that is consistent with $\sigma(F)$ also contains vertex $v$. The local diagnosability of a vertex $v$ in $G$ is defined to be the maximum integer of $t$ such that $G$ is $t^{*}$-diagnosable at the vertex $v$. The relationship between diagnosability and local diagnosability is revealed in the following theorems.

Theorem 3: [16] A graph $G$ is $t$-diagnosable iff it is $t^{*}$-diagnosable at each vertex.

Theorem 4: [16] Let $G$ denote the underlying topology of a multiprocessor system. Then $\tau(G)=\min \left\{\tau_{G}^{*}(v) \mid v \in V(G)\right\}$.

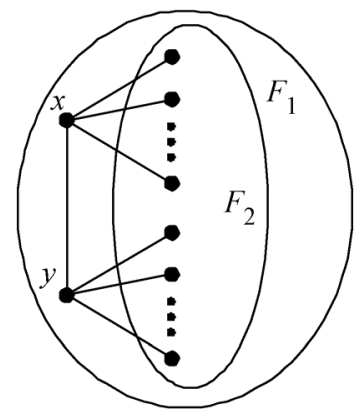

Fig. 2. A $t$-connected network is not conditionally $2 t$-diagnosable.

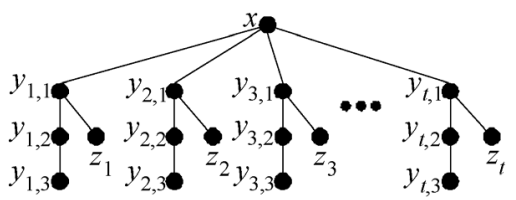

Fig. 3. A wind-bell-tree $W B_{G}(x ; t)$.

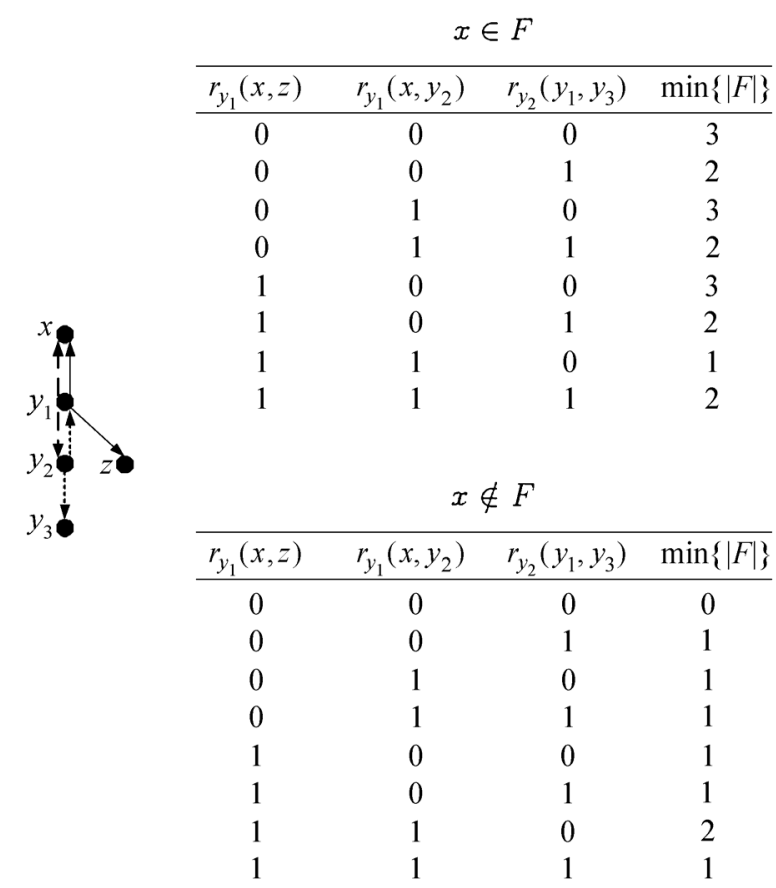

Fig. 4. Illustrations for Proposition 1.

\section{Conditional Fault Diagnosis}

In [20], Lai et al. proposed the concept of conditional fault diagnosis by restricting that, for each processor $v$ in the network, all the processors which are directly connected to $v$ do not fail at the same time. Recently, Hsieh and Chuang [13], [15] proposed the concept of strong diagnosability on regular networks and product networks. A system is said to be stronglyt-diagnosable if it is $t$-diagnosable, and can achieve $(t+1)$-diagnosable, except for the case that the neighbors of a processor fail simultaneously.

Suppose that $G=(V, E)$. A set $F \subset V(G)$ is a conditional faulty set if $N_{G}(v) \nsubseteq F$ for any vertex $v \in V(G)-F$. A system $G$ is conditionally faulty if the faulty vertex set of $G$ forms a conditional faulty set. For any two distinct conditional faulty sets $F_{1}$ and $F_{2}$ of $G$ with $\left|F_{1}\right| \leq t$ and $\left|F_{2}\right| \leq t$, if $\left(F_{1}, F_{2}\right)$ 


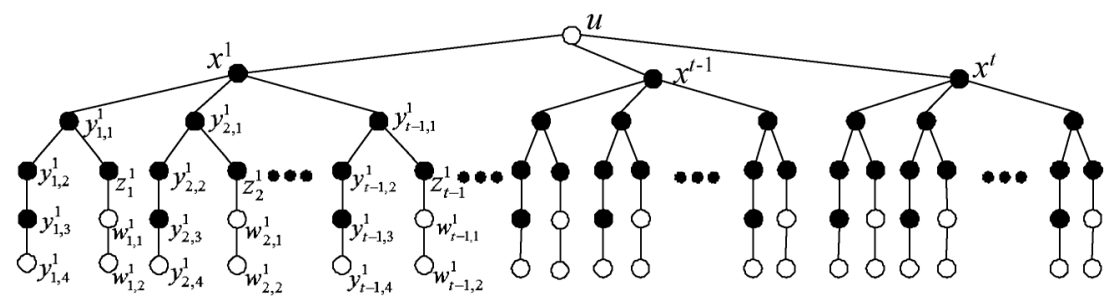

Fig. 5. A balanced wind-bell-tree $B W B_{G}(u ; t)$.

is a distinguishable pair, then $G$ is conditionally t-diagnosable. The maximum number of conditional faulty vertices that can be correctly identified in $G$ is called the conditional diagnosability of $G$. Let $v$ be any vertex in $G$. The conditional local diagnosability of $v$ in $G$ is defined to be the maximum integer of $t$ such that $G$ is conditionally $t^{*}$-diagnosable at vertex $v$. It is trivial that $\tau^{c}(G) \geq \tau(G)$ and $\tau^{c}(G)=\min \left\{\tau_{G}^{c}(v) \mid v \in V(G)\right\}$. Now we give an example to show that a $t$-connected network is not conditionally $2 t$-diagnosable. Let $G$ be a $t$-connected network with two adjacent vertices $x$ and $y$, where $\operatorname{deg}(x)=t$ and $\operatorname{deg}(y)=t$. We set $F_{1}=N_{G}(x) \cup N_{G}(y)$ and $F_{2}=$ $\left(N_{G}(x) \cup N_{G}(y)\right)-\{x, y\}$. See Fig. 2 for an illustration. By Theorem $1, F_{1}$ and $F_{2}$ are indistinguishable. Thus, $G$ is not conditionally $2 t$-diagnosable.

In Section IV, we propose a specific structure named the balanced wind-bell-tree, and give an algorithm to identify whether a given vertex is in a state of fault-free or fault in a conditional faulty system under the MM model. With our algorithm, a vertex can be identified correctly if the total number of faulty vertices does not exceed $2 t-1$, where $t$ is the connectivity of the system.

\section{A Local Diagnosis Algorithm}

Chiang and Tan proposed a local diagnosis algorithm called the extended star in [7]. In this section, we propose another specific structure, the wind-bell-tree, for local diagnosis, and give a local diagnosis algorithm under the MM model. Different with the extended star, we can construct a specific structure for conditional fault diagnosis using the wind-bell-tree. We describe the conditional local diagnosis algorithm in Section IV. Now, we give the definition of a wind-bell-tree as follows.

Definition 1: Let $G=(V, E)$ be a graph, and let $x$ be a vertex in $G$. A wind-bell-tree of order $t$ rooted at $x$ is defined to be the subgraph of $G$, denoted by $W B_{G}(x ; t)$, such that $V\left(W B_{G}(x ; t)\right)=\{x\} \cup\left\{y_{i, j}, z_{i} \mid 1 \leq i \leq t, 1 \leq j \leq 3\right\}$, and $E\left(W B_{G}(x ; t)\right)=\left\{\left\{x, y_{i, 1}\right\},\left\{y_{i, k}, y_{i, k+1}\right\},\left\{y_{i, 1}, z_{i}\right\} \mid 1 \leq\right.$ $i \leq t, 1 \leq k \leq 2\}$. Fig. 3 illustrates the $W B_{G}(x ; t)$.

Proposition 1: Let $V\left(W B_{G}(x ; 1)\right)=\left\{x, y_{1}, y_{2}, y_{3}, z\right\}$ and $E\left(W B_{G}(x ; 1)\right)=\left\{\left(x, y_{1}\right),\left(y_{1}, y_{2}\right),\left(y_{2}, y_{3}\right),\left(y_{1}, z\right)\right\}$ as illustrated in Fig. 4. Suppose that $F$ is a faulty set, and $F \subseteq V\left(W B_{G}(x ; 1)\right)$. Depending on the definition of the MM model and the faulty or fault-free status of $x$, the relation between the result of $\left(r_{y_{1}}(x, z), r_{y_{1}}\left(x, y_{2}\right), r_{y_{2}}\left(y_{1}, y_{3}\right)\right)$ and the least number of $|F|$ is illustrated in Fig. 4.

Let $G$ be a graph, and $F$ be a faulty set in $G$ with $|F| \leq t$. We propose the algorithm LDA (Local-Diagnosis-Algorithm) to identify the faulty or fault-free status of a vertex $x$ in a windbell-tree $W B_{G}(x ; t)$ under the MM model, and prove the correctness of the algorithm LDA in Theorem 5.

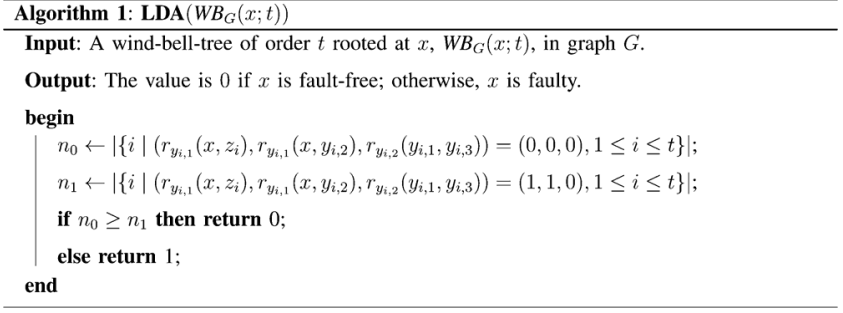

Theorem 5: Let $W B_{G}(x ; t)$ be a wind-bell-tree of order $t$ rooted at $x$. If $F$ is a faulty set in $G$ with $|F| \leq t$, then the faulty or fault-free status of vertex $x$ can be identified correctly with the algorithm $\mathbf{L D A}\left(W B_{G}(x ; t)\right)$.

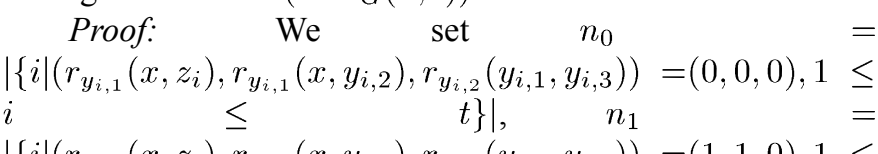
$\mid\left\{i \mid\left(r_{y_{i, 1}}\left(x, z_{i}\right), r_{y_{i, 1}}\left(x, y_{i, 2}\right), r_{y_{i, 2}}\left(y_{i, 1}, y_{i, 3}\right)\right)=(1,1,0), 1 \leq\right.$ $i \leq t\} \mid$, and $n_{2}=t-n_{0}-n_{1}$. In the algorithm LDA $\left(W B_{G}(x ; t)\right)$, we claim that $x \notin F$ if $n_{0} \geq n_{1}$, and $x \in F$ if $n_{0}<n_{1}$. We prove the theorem by contradiction. Assume that $x \in F$, and $n_{0} \geq n_{1}$. By Proposition 1, we have $|F| \geq 2 n_{0}+n_{2}+1 \geq\left(n_{0}+n_{1}\right)+n_{2}+1=t+1$, which contradicts the assumption that $|F| \leq t$. Now we assume that $x \notin F$, and $n_{0}<n_{1}$. By Proposition 1, we have $|F| \geq 2 n_{1}+n_{2} \geq\left(n_{0}+n_{1}+1\right)+n_{2}=t+1$, which contradicts the assumption that $|F| \leq t$. Thus the theorem holds.

\section{A Conditional Local Diagnosis Algorithm}

In this section, we discuss the local diagnosability for a multiprocessor system with a conditional faulty set. We propose a specific structure, called a balanced wind-bell-tree, for conditional local diagnosis. The definition of a balanced wind-belltree is described as follows.

Definition 2: Let $G=(V, E)$ be a graph, $u \in V(G)$, and $t \geq 2$. A balanced wind-bell-tree of order $t$ rooted at $u$ is defined to be the subgraph of $G$, denoted by $B W B_{G}(u ; t)$, such that $V\left(B W B_{G}(u ; t)\right)=\{u\} \cup$ $\left\{x^{i}, y_{j, k}^{i}, z_{j}^{i}, w_{j, 1}^{i}, w_{j, 2}^{i} \mid 1 \leq i \leq t, 1 \leq j \leq t-1,1 \leq k \leq 4\right\}$, and $E\left(B W B_{G}(u ; t)\right)=\left\{\left\{u, x^{i}\right\},\left\{x^{i}, y_{j, 1}^{i}\right\},\left\{y_{j, k}^{i}, y_{j, k+1}^{i}\right\}\right.$, $\left\{y_{j, 1}^{i}, z_{j}^{i}\right\},\left\{z_{j}^{i}, w_{j, 1}^{i}\right\},\left\{w_{j, 1}^{i}, w_{j, 2}^{i}\right\} \mid 1 \leq i \leq t, 1 \leq j \leq$ $t-1,1 \leq k \leq 3\}$. Fig. 5 illustrates the $B W B_{G}(u ; t)$.

Let $G$ be a graph, and $F$ be a conditional faulty set in $G$ with $|F| \leq 2 t-1$. We propose the algorithm CFLDA (ConditionalFault-Local-Diagnosis-Algorithm) to identify the faulty status of a vertex $u$ in a balanced wind-bell-tree $B W B_{G}(u ; t)$ with the conditionally faulty set $F$ under the MM model. 


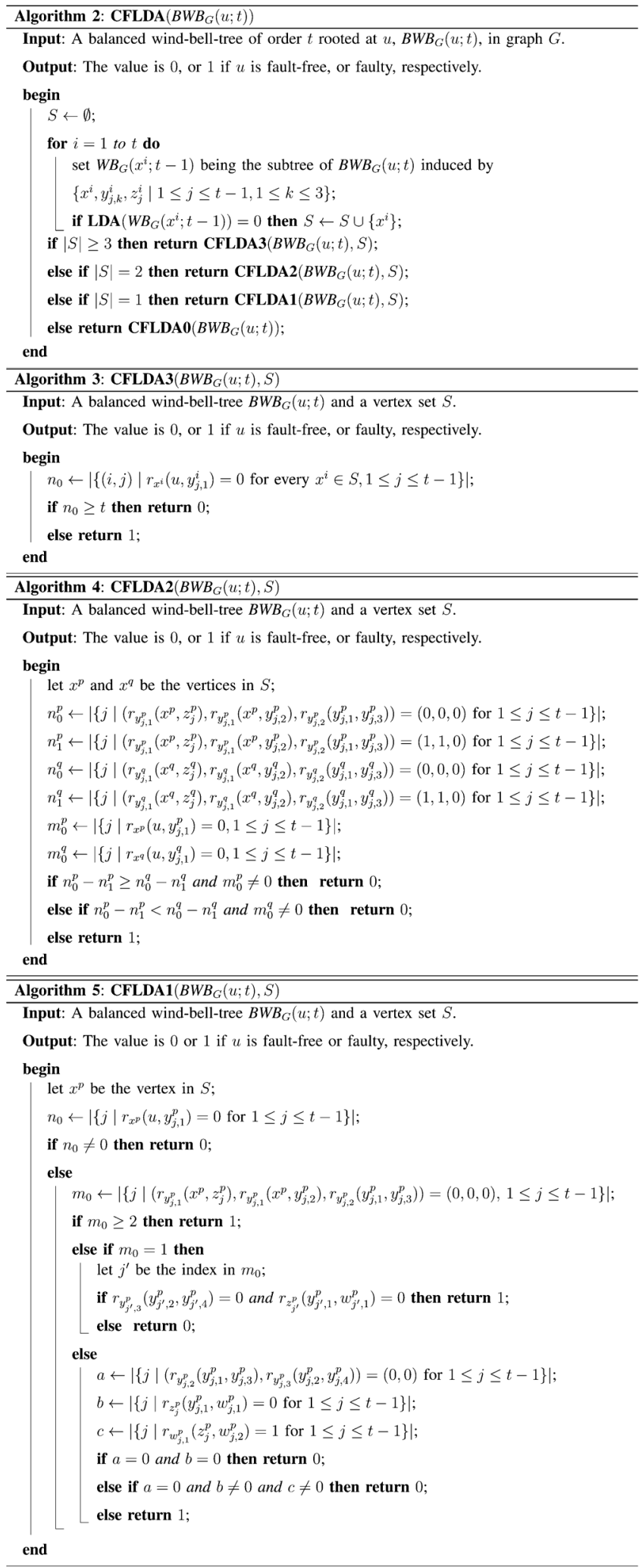

We need the following lemma for the later proof of our results.

Lemma 1: Suppose that $F$ is a conditional faulty set in $B W B_{G}(u ; t)$ with $|F| \leq 2 t-1$. Let

$$
\begin{aligned}
& A=\left\{x^{i} \mid \mathbf{L D A}\left(W B_{G}\left(x^{i} ; t-1\right)\right)=0, x^{i} \in F\right\}, \text { and } \\
& B=\left\{x^{i} \mid \mathbf{L D A}\left(W B_{G}\left(x^{i} ; t-1\right)\right)=1, x^{i} \notin F\right\} .
\end{aligned}
$$
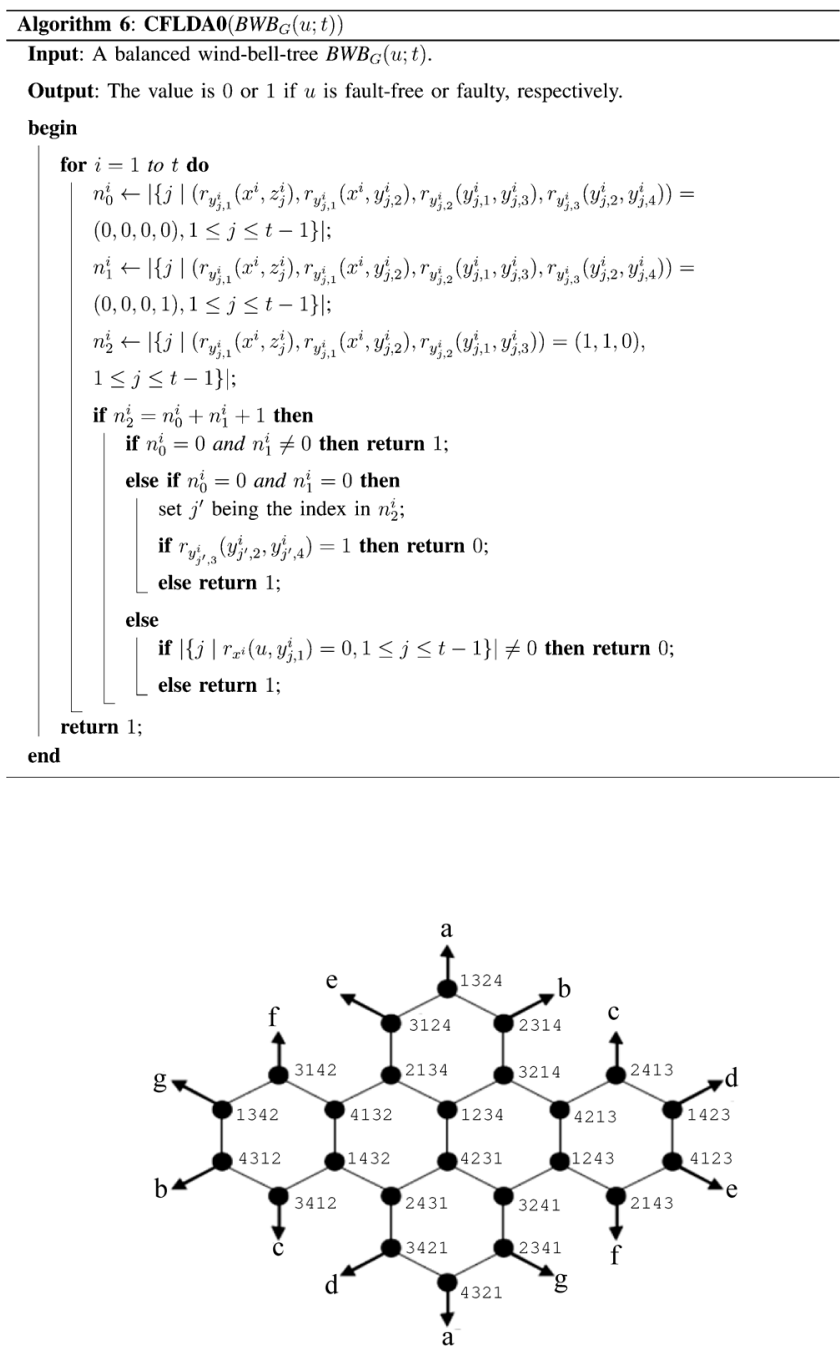

Fig. 6. A star graph $S_{4}$; the same letters represent the connected edges.

Then $|A|+|B| \leq 1$. That is, for $1 \leq i \leq t$, there is at most one mistake in diagnosis of $x^{i}$ with the algorithm LDA.

Proof: We prove the lemma by contradiction. Assume that $|A|+|B| \geq 2$. By Theorem 5, we have $|F| \geq 2 t>2 t-1$, which contradicts the assumption that $|F| \leq 2 t-1$.

Lemma 2: Let $t \geq 5$, and $S=\left\{x^{i} \mid \mathbf{L D A}\left(W B_{G}\left(x^{i} ; t-\right.\right.\right.$ 1)) $=0\}$. Suppose that $F$ is a conditional faulty set in $G$ with $|F| \leq 2 t-1$. Then the faulty or fault-free status of vertex $u$ can be identified correctly by the algorithms CFLDA3 $\left(B W B_{G}(u ; t), S\right)$, CFLDA2 $\left(B W B_{G}(u ; t), S\right), \quad$ CFLDA1 $\left(B W B_{G}(u ; t), S\right)$, and CFLDA0 $\left(B W B_{G}(u ; t), S\right)$ if $|S| \geq 3,|S|=2,|S|=1$, and $|S|=0$, respectively.

The proof of Lemma 2 is presented in the Appendix. By Lemma 2, we can prove the correctness of the algorithms CFLDA3, CFLDA2, CFLDA1, and CFLDA0. Hence, we have the following theorem.

Theorem 6: Suppose that $t \geq 5$. Let $B W B_{G}(u ; t)$ be a balanced wind-bell-tree of order $t$ rooted at $u$. If $F$ is a conditional faulty set in $G$ with $|F| \leq 2 t-1$, then the faulty or fault-free status of vertex $u$ can be identified correctly with the algorithm CFLDA $\left(B W B_{G}(u ; t)\right)$. 


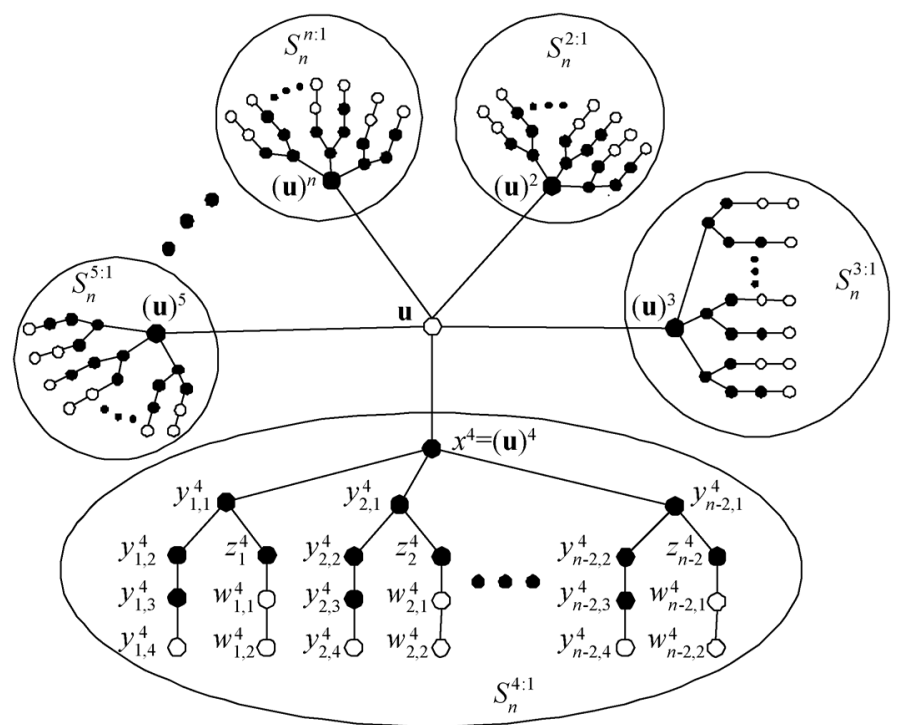

$$
\begin{aligned}
& y_{1,1}^{4}=\left((\mathbf{u})^{4}\right)^{5} \\
& y_{1,2}^{4}=\left(\left((\mathbf{u})^{4}\right)^{5}\right)^{2} \quad z_{1}^{4}=\left(\left((\mathbf{u})^{4}\right)^{5}\right)^{3} \\
& y_{1,3}^{4}=\left(\left(\left((\mathbf{u})^{4}\right)^{5}\right)^{2}\right)^{3} \quad w_{1,1}^{4}=\left(\left(\left((\mathbf{u})^{4}\right)^{5}\right)^{3}\right)^{6} \\
& y_{1,4}^{4}=\left(\left(\left(\left((\mathbf{u})^{4}\right)^{5}\right)^{2}\right)^{3}\right)^{6} \quad w_{1,2}^{4}=\left(\left(\left(\left((\mathbf{u})^{4}\right)^{5}\right)^{3}\right)^{6}\right)^{2} \\
& y_{2,1}^{4}=\left((\mathbf{u})^{4}\right)^{6} \\
& y_{2,2}^{4}=\left(\left((\mathbf{u})^{4}\right)^{6}\right)^{2} \quad z_{2}^{4}=\left(\left((\mathbf{u})^{4}\right)^{6}\right)^{3} \\
& y_{2,3}^{4}=\left(\left(\left((\mathbf{u})^{4}\right)^{6}\right)^{2}\right)^{3} \quad w_{2,1}^{4}=\left(\left(\left((\mathbf{u})^{4}\right)^{6}\right)^{3}\right)^{5} \\
& y_{2,4}^{4}=\left(\left(\left(\left((\mathbf{u})^{4}\right)^{6}\right)^{2}\right)^{3}\right)^{5} \quad w_{2,2}^{4}=\left(\left(\left(\left((\mathbf{u})^{4}\right)^{6}\right)^{3}\right)^{5}\right)^{2} \\
& \text { ! } \\
& y_{n-2,1}^{4}=\left((\mathbf{u})^{4}\right)^{3} \\
& y_{n-2,2}^{4}=\left(\left((\mathbf{u})^{4}\right)^{3}\right)^{2} \quad z_{n-2}^{4}=\left(\left((\mathbf{u})^{4}\right)^{3}\right)^{5} \\
& y_{n-2,3}^{4}=\left(\left(\left((\mathbf{u})^{4}\right)^{3}\right)^{2}\right)^{5} \quad w_{n-2,1}^{4}=\left(\left(\left((\mathbf{u})^{4}\right)^{3}\right)^{5}\right)^{6} \\
& y_{n-2,4}^{4}=\left(\left(\left(\left((\mathbf{u})^{4}\right)^{3}\right)^{2}\right)^{5}\right)^{6} \quad w_{n-2,2}^{4}=\left(\left(\left(\left((\mathbf{u})^{4}\right)^{3}\right)^{5}\right)^{6}\right)^{2}
\end{aligned}
$$

Fig. 7. An illustration for constructing a balanced wind-bell-tree $B W B_{S_{n}}(\mathbf{u} ; n-1)$ in a star graph $S_{n}$.

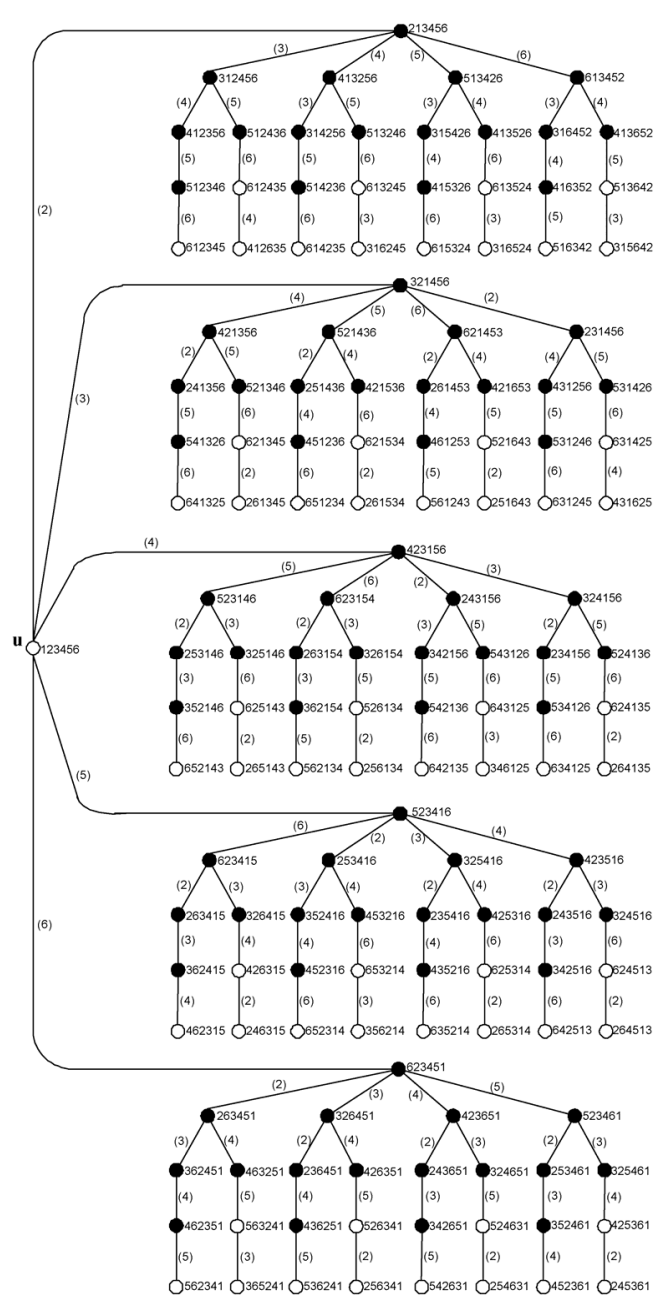

Fig. 8. $B W B_{S_{6}}(\mathbf{u} ; 5)$. The number $i$ in the parentheses on each edge represents the $i$-dimensional edge in $S_{6}$.

\section{APPLICATION ON THE STAR GRAPH}

In this section, we show that the proposed diagnosis algorithm CFLDA can be applied to the star graph, the well-known interconnection network of multiprocessor systems. The star graph is proposed in [1]. The $n$-dimensional star graph is an attractive alternative to the $n$-cube topology for interconnecting processors in parallel computers and distributed systems because of its recursive structure, and vertex and edge symmetry. The star graphs are able to embed some well-known network topologies, such as trees [2], grids [19], hypercubes [27], and cycles [31]. Many efficient communication algorithms on star graphs for broadcasting, gossiping, scattering, and Fourier transform are also proposed in [4], [10], [11], [26]. The vertex set $V$ of an $n$-dimensional star graph $S_{n}$ is $\left\{u_{1} u_{2} \ldots u_{n} \mid u_{1} u_{2} \ldots u_{n}\right.$ is a permutation of $1,2, \ldots, n\}$. Thus $\left|V\left(S_{n}\right)\right|=n$ ! The adjacency is defined as follows; $u_{1} u_{2} \ldots u_{i} \ldots u_{n}$ is adjacent to $v_{1} v_{2} \ldots v_{i} \ldots v_{n}$ through an edge of dimension $i$ with $2 \leq i \leq$ $n$ if $v_{j}=u_{j}$ for $j \notin\{1, i\}, v_{1}=u_{i}$, and $v_{i}=u_{1}$. Hence the degree of every vertex in $S_{n}$ is $n-1$. For example, in a $S_{4}$ containing 4 ! vertices, two vertices 1234 and 4231 are neighbors, and joined through an edge labeled 4. Fig. 6 illustrates the $S_{4}$. Let $\mathbf{u}=u_{1} u_{2} \ldots u_{n}$ be any vertex of $S_{n}$. By the definition of $S_{n}$, there is exactly one neighbor $\mathbf{v}$ of $\mathbf{u}$ such that $\mathbf{u}$ and $\mathbf{v}$ are adjacent through an $i$-dimensional edge with $2 \leq i \leq n$. Now we propose the following algorithm called B-IN-S to construct a balanced wind-bell-tree in a star graph $S_{n}$ for $n \geq 6$ with $\left|V\left(S_{n}\right)\right| \geq\left|V\left(B W B_{G}(u ; t)\right)\right|$. Let $(\mathbf{u})^{i}$ be the vertices $x^{i}$ in a balanced wind-bell-tree $B W B_{S_{n}}(\mathbf{u} ; n-1)$ for every $2 \leq i \leq n$. Thus every subtree of $B W B_{S_{n}}(\mathbf{u} ; n-1)$ induced by $\left\{x^{i}, y_{j, k}^{i}, z_{j}^{i}, w_{j, 1}^{i}, w_{j, 2}^{i} \mid 2 \leq i \leq n, 1 \leq j \leq n-2,1 \leq k \leq 4\right\}$ is located in mutually distinct components of $S_{n}$. See Fig. 7 for an illustration. With this concept, we can construct a balanced wind-bell-tree in a star graph by the algorithm B-IN-S. We describe a $B W B_{S_{6}}(\mathbf{u} ; 5)$ constructed by the algorithm B-IN-S in Fig. 8.

\section{CONCLUding REMARKS}

The issue of identifying faulty processors is important for the design of multiprocessor systems, which are implementable with VLSI. The process of identifying all the faulty processors is called system-level diagnosis. Under the MM model, each 


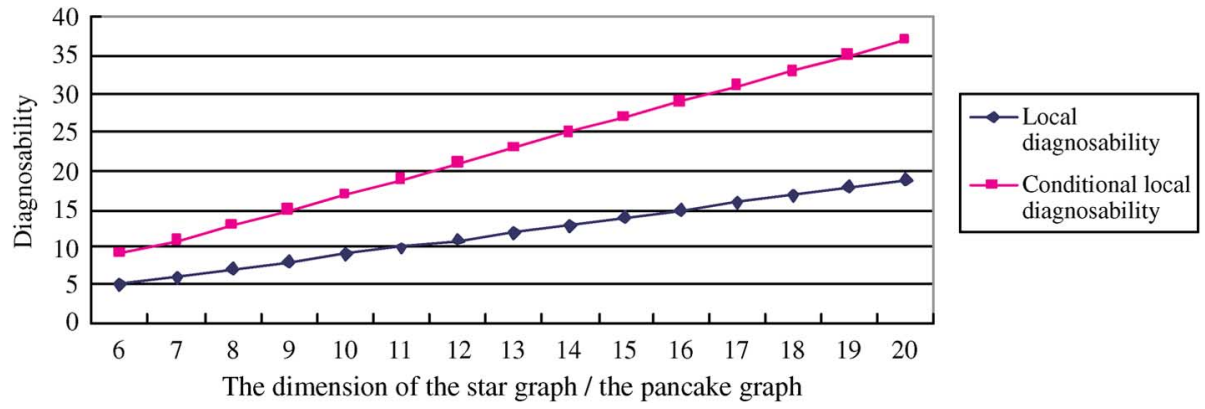

Fig. 9. The local diagnosability and conditional local diagnosability for a vertex in the star graph or in the pancake graph.

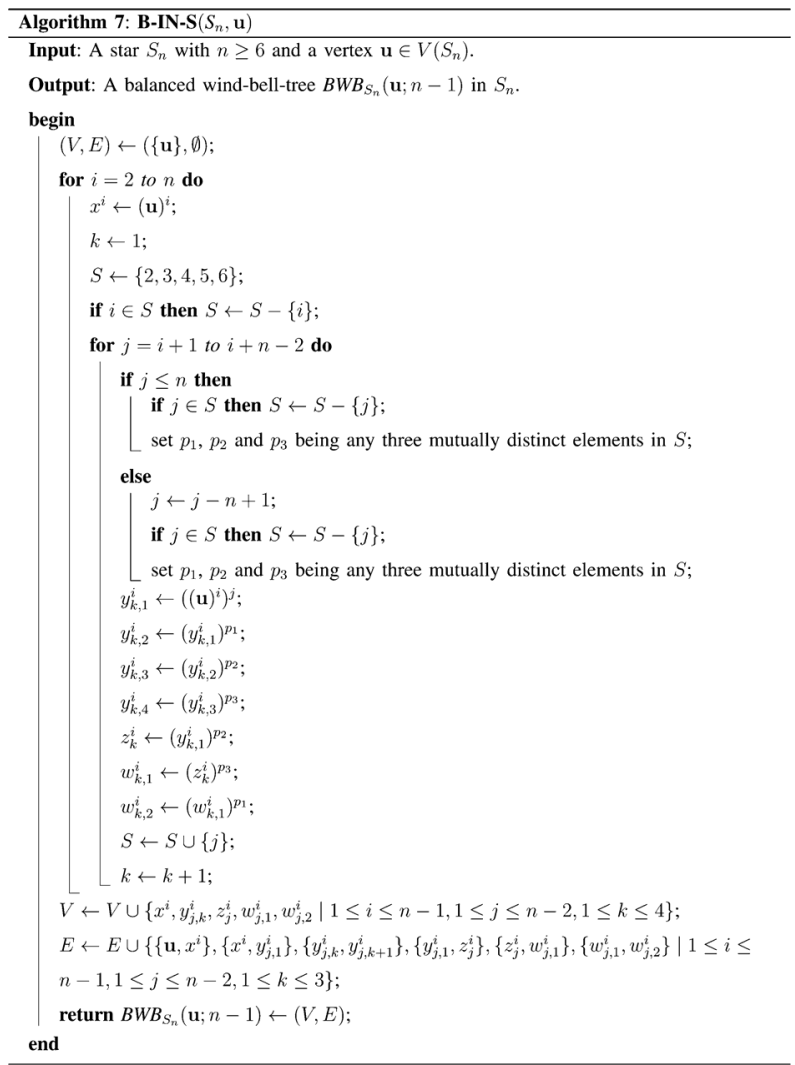

processor acts as a comparator to test each pair of adjacent two processors. Sengupta and Dahbura [30] proposed a polynomial-time algorithm with time complexity $O\left(N^{5}\right)$ to diagnose a system with a total number $N$ of processors under the MM model. In the random-fault probabilistic model of multiprocessor systems, processors are assumed to fail statistically independently. For many practical multiprocessor systems or interconnection networks, the probability that all the neighbors of a processor are faulty simultaneously is very small. Thus, we address the conditional local diagnosability problem under the MM model in this paper. We propose a specific structure called the balanced wind-bell-tree, and give an algorithm CFLDA to diagnose a vertex in the system with a conditional faulty set under the MM model. According to our results, a specific $t$-connected network with $N$ processors is conditionally $(2 t-1)^{*}$-diagnosable. In Section $\mathrm{V}$, we give an application by constructing the balanced wind-bell-tree in an $n$-dimensional star graph $S_{n}$. Akers and Krishnameurthy [1] proposed another family of interesting interconnection networks, called the pancake graph. Sim-
TABLE I

The Time Complexity and Diagnosability in a System $G$ Under the MM Model AsSuming THAT $|V(G)|=N$, AND $\delta(G)=t$

\begin{tabular}{|c|c|c|}
\hline Algorithm & Time complexity & Diagnosability \\
\hline Sengupta and Dahbura [30] & $O\left(N^{5}\right)$ & $t$ \\
\hline LDA $\left(W B_{G}(x ; t)\right)$ & $O(N \log N)$ & $t$ \\
\hline CFLDA $\left(B W B_{G}(u ; t)\right)$ & $O\left(N(\log N)^{2}\right)$ & Conditionally $2 t-1$ \\
\hline
\end{tabular}

ilar to $S_{n}$, the $n$-dimensional pancake graph is an $(n-1)$-regular graph with $n$ ! vertices. Moreover, the pancake graph is vertex transitive. With an algorithm similar to B-IN-S, a balanced wind-bell-tree can be constructed in a pancake graph. Fig. 9 shows that under the same dimension, the conditional local diagnosability for a vertex is about twice larger than the local diagnosability for a vertex in the star graph or in the pancake graph.

Now, we measure the time complexity of the proposed algorithm CFLDA. Many practical systems with $N$ vertices have degrees on the order of $O(\log N)$ for each vertex. For a system $G$, a balanced wind-bell-tree $B W B_{G}(u ; \log N)$ of order $\log N$ rooted at $u$ can be constructed with time complexity $O\left((\log N)^{2}\right)$. The time complexity of the algorithm LDA is $O(\log N)$ for $W B_{G}\left(x^{i} ; \log N-1\right)$, and it runs $O(\log N)$ times. As a result, the time complexity of the CFLDA algorithm is $O\left((\log N)^{2}\right)$. Consequently, the total time for diagnosing all the faulty vertices is $O\left(N(\log N)^{2}\right)$. Table I shows the time complexity and diagnosability in some algorithms under the MM model. The $n$-dimensional star graph $S_{n}$ has $n$ ! vertices, and the degree of each vertex is $O(n)$. Let $N=n$ !. We have $O(n)=O(\log N / \log n)=O(\log N / \log \log N)$. Thus the time complexity to diagnose all the faulty vertices in a star graph is $O\left(N(\log N /(\log \log N))^{2}\right)$.

Future works will try to find some specific structure for the existing practical multiprocessor systems and interconnection networks. Then we propose to design the efficient diagnosis algorithm, and prove the diagnosability of the system with this useful structure in accordance with various conditions and diagnosis models.

\section{APPENDIX}

Proof of Lemma 2:

Proof: We consider the following cases. 
Case 1) Suppose that $|S| \geq 3$. We prove the correctness of the algorithm CFLDA3 $\left(B W B_{G}(u ; t), S\right)$ as follows. We set $n_{0}=\mid\left\{(i, j) \mid r_{x^{i}}\left(u, y_{j, 1}^{i}\right)=0\right.$ for every $\left.x^{i} \in S, 1 \leq j \leq t-1\right\} \mid, n_{1}=$ $\mid\left\{(i, j) \mid r_{x^{i}}\left(u, y_{j, 1}^{i}\right)=1\right.$ for every $x^{i} \in S, 1 \leq j \leq$ $t-1\} \mid$, and $Y=\left\{y_{j .1}^{i} \mid x^{i} \in S-F, 1 \leq j \leq t-1\right\}$. In the algorithm CFLDA3 $\left(B W B_{G}(u ; t), S\right)$, we claim that $u \notin F$ if $n_{0} \geq t$, and $u \in F$ if $n_{0}<t$. By Lemma 1, $|S \cap F| \leq 1$. We have the following subcases.

Subcase 1.1: Suppose that $u \notin F$, and $|S \cap F|=$ 0 . Thus $|F \cap Y|=n_{1} \leq(2 t-1)-(t-|S|)=$ $t+|S|-1$. Because $n_{0}+n_{1}=|S|(t-1)$, we have $n_{0} \geq|S|(t-1)-(t+|S|-1) \geq t$ if $t \geq 5$, and $t \geq|S| \geq 3$.

Subcase 1.2: Suppose that $u \notin F$ and $|S \cap F|=$ 1. Thus $|F \cap Y| \leq(2 t-1)-(t-|S|)-t=$ $|S|-1$. Because $n_{0}+n_{1}=|S|(t-1)$, we have $n_{0} \geq|S|(t-1)-(|S|-1)-(t-1) \geq t$ if $t \geq 5$, and $t \geq|S| \geq 3$.

Subcase 1.3: Suppose that $u \in F$. Because $\mid S \cap$ $F \mid \leq 1$, we have $n_{0} \leq t-1$.

Thus, the algorithm CFLDA3 $\left(B W B_{G}(u ; t), S\right)$ is correct.

Case 2) Suppose that $|S|=2$. We prove the correctness of the algorithm CFLDA2 $\left(B W B_{G}(u ; t), S\right)$ as follows. Let $S=\left\{x^{p}, x^{q}\right\}$. For each $1 \leq j \leq t-1$, suppose that

$$
\begin{aligned}
& n_{0}^{p}=\mid\left\{j \mid\left(r_{y_{j, 1}^{p}}\left(x^{p}, z_{j}^{p}\right), r_{y_{j, 1}^{p}}\left(x^{p}, y_{j, 2}^{p}\right), r_{y_{j, 2}^{p}}\left(y_{j, 1}^{p}, y_{j, 3}^{p}\right)\right)\right. \\
& =(0,0,0)\} \mid \text {, } \\
& n_{1}^{p}=\mid\left\{j \mid\left(r_{y_{j, 1}^{p}}\left(x^{p}, z_{j}^{p}\right), r_{y_{j, 1}^{p}}\left(x^{p}, y_{j, 2}^{p}\right), r_{y_{j, 2}^{p}}\left(y_{j, 1}^{p}, y_{j, 3}^{p}\right)\right)\right. \\
& =(1,1,0)\} \mid \text {, } \\
& n_{0}^{q}=\mid\left\{j \mid\left(r_{y_{j, 1}^{q}}\left(x^{q}, z_{j}^{q}\right), r_{y_{j, 1}^{q}}\left(x^{q}, y_{j, 2}^{q}\right), r_{y_{j, 2}^{q}}\left(y_{j, 1}^{q}, y_{j, 3}^{q}\right)\right)\right. \\
& =(0,0,0)\} \mid \text {, and } \\
& n_{1}^{q}=\mid\left\{j \mid\left(r_{y_{j, 1}^{q}}\left(x^{q}, z_{j}^{q}\right), r_{y_{j, 1}^{q}}\left(x^{q}, y_{j, 2}^{q}\right), r_{y_{j, 2}^{q}}\left(y_{j, 1}^{q}, y_{j, 3}^{q}\right)\right)\right. \\
& =(1,1,0)\} \mid \text {. }
\end{aligned}
$$

We claim that if $n_{0}^{p}-n_{1}^{p} \geq n_{0}^{q}-n_{1}^{q}$, then $x^{p} \notin F$. We prove by contradiction. Assume that $x^{p} \in F$, and $n_{0}^{p}-n_{1}^{p} \geq n_{0}^{q}-n_{1}^{q}$. By Lemma $1,\left|\left\{x^{p}, x^{q}\right\} \cap F\right| \leq 1$, hence $x^{q} \notin F$. By Proposition 1, we have $|F| \geq$ $2 n_{0}^{p}+\left((t-1)-n_{0}^{p}-n_{1}^{p}\right)+2 n_{1}^{q}+\left((t-1)-n_{0}^{q}-n_{1}^{q}\right)+$ $(t-2)+1=\left(n_{0}^{p}-n_{1}^{p}\right)-\left(n_{0}^{q}-n_{1}^{q}\right)+(3 t-3)+1>$ $2 t-1$ if $t \geq 5$, which contradicts the assumption that $|F| \leq 2 t-1$. Similarly, we have $x^{q} \notin F$ if $n_{0}^{p}-n_{1}^{p}<n_{0}^{q}-n_{1}^{q}$.

Without loss of generality, we assume that $n_{0}^{p}-n_{1}^{p} \geq$ $n_{0}^{q}-n_{1}^{q}$, and $x^{p} \notin F$. Obviously, if there exists some $j$ such that $r_{x^{p}}\left(u, y_{j, 1}^{p}\right)=0$, then $u \notin F$. Now we prove that if $r_{x^{p}}\left(u, y_{j, 1}^{p}\right)=1$ for every $1 \leq j \leq$ $t-1$, then $u \in F$. Let $Y_{p}=\left\{y_{j, 1}^{p} \mid 1 \leq j \leq t-1\right\}$. We claim that $Y_{p} \nsubseteq \subset F$ if $n_{0}^{p}-n_{1}^{p} \geq n_{0}^{q}-n_{1}^{q}$. We prove by contradiction. Assume that $Y_{p} \subseteq F$, and $x^{p} \notin F$. Thus we have $\left|Y_{p} \cap F\right|=t-1$. That is, $\left|V\left(W B_{G}\left(x^{p} ; t-1\right)\right) \cap F\right|=t-1$. Hence $\left(r_{y_{j, 1}^{p}}\left(x^{p}, z_{j}^{p}\right), r_{y_{j, 1}^{p}}\left(x^{p}, y_{j, 2}^{p}\right), r_{y_{j, 2}^{p}}\left(y_{j, 1}^{p}, y_{j, 3}^{p}\right)\right) \in$ $\{(0,1,0),(1,1,0)\}$ for every $1 \leq j \leq t-1$. Thus we have $n_{0}^{p}=n_{1}^{p}=0$. Because $|F| \leq 2 t-1$, we have $\left|V\left(W B_{G}\left(x^{q} ; t-1\right)\right) \cap F\right| \leq(2 t-1)-$ $(t-1)-(t-2)=2$. By Theorem 5, $x^{q} \notin F$. Hence $n_{1}^{q} \leq 1$, and $n_{0}^{q} \geq t-3$. Thus, $n_{0}^{q}-n_{1}^{q} \geq$ $t-4>n_{0}^{p}-n_{1}^{p}=0$ for $t \geq 5$, which contradicts the assumption that $n_{0}^{p}-n_{1}^{p} \geq n_{0}^{q}-n_{1}^{q}$. Hence, we have $Y_{p} \nsubseteq \mp$. Therefore, if $r_{x^{p}}\left(u, y_{j, 1}^{p}\right)=1$ for every $1 \leq j \leq t-1$, then $u \in F$. Thus the algorithm CFLDA2 $\left(B W B_{G}(u ; t), S\right)$ is correct.

Case 3) Suppose that $|S|=1$. We prove the correctness of the algorithm CFLDA1 $\left(B W B_{G}(u ; t), S\right)$ as follows. We set $S=\left\{x^{p}\right\}$. First, we claim that $x^{p} \notin F$. We prove it by contradiction. Assume that $x^{p} \in F$. Because $N_{G}\left(x^{p}\right) \subseteq F$, we have $u \in F$. For $1 \leq j \leq t-1$, let $m_{0}=$ $\mid\left\{j \mid\left(r_{y_{j, 1}^{p}}\left(x^{p}, z_{j}^{p}\right), r_{y_{j, 1}^{p}}\left(x^{p}, y_{j, 2}^{p}\right), r_{y_{j, 2}^{p}}\left(y_{j, 1}^{p}, y_{j, 3}^{p}\right)\right)=\right.$ $(0,0,0)\} \mid$ and $m_{1}=$ $\mid\left\{j \mid\left(r_{y_{j, 1}^{p}}\left(x^{p}, z_{j}^{p}\right), r_{y_{j, 1}^{p}}\left(x^{p}, y_{j, 2}^{p}\right), r_{y_{j, 2}^{p}}\left(y_{j, 1}^{p}, y_{j, 3}^{p}\right)\right)=\right.$ $(1,1,0)\} \mid$. By $\quad$ Proposition 1, $|F| \geq 2 m_{0}+\left(t-1-m_{0}-m_{1}\right)+(t-1)+1+$ $1=2 t+\left(m_{0}-m_{1}\right)>2 t-1$, which contradicts the assumption that $|F| \leq 2 t-1$. Therefore, we have $x^{p} \notin F$. Obviously, if there exists some $j$ such that $r_{x^{p}}\left(u, y_{j, 1}^{p}\right)=0$, then $u \notin F$. Suppose that $r_{x^{p}}\left(u, y_{j, 1}^{p}\right)=1$ for every $1 \leq j \leq t-1$. We have the following subcases.

Subcase 3.1: If $m_{0} \geq 2$, then $u \in F$. Assume that $u \notin F$ by contradiction. If there exists some $j^{\prime}$ such that $\left(r_{y_{j^{\prime}, 1}^{p}}\left(x^{p}, z_{j^{\prime}}^{p}\right), r_{y_{j^{\prime}, 1}^{p}}\left(x^{p}, y_{j^{\prime}, 2}^{p}\right)\right.$, $\left.r_{y_{j^{\prime}, 2}^{p}}\left(y_{j^{\prime}, 1}^{p}, y_{j^{\prime}, 3}^{p}\right)\right)=(0,0,0)$, then the two vertices $y_{j^{\prime}, 1}^{p}$ and $y_{j^{\prime}, 2}^{p}$ are faulty. By Proposition 1 , we have $|F| \geq 2 m_{0}+2 m_{1}+\left(t-1-m_{0}-m_{1}\right)+$ $(t-1)=2 t-2+m_{0}+m_{1} \geq 2 t>2 t-1$, which contradicts the assumption that $|F| \leq 2 t-1$.

Subcase 3.2: Suppose that $m_{0}=1$. Let $j^{\prime}$ be an index such that $\left(r_{y_{j^{\prime}, 1}^{p}}\left(x^{p}, z_{j^{\prime}}^{p}\right), r_{y_{j^{\prime}, 1}^{p}}\left(x^{p}, y_{j^{\prime}, 2}^{p}\right)\right.$, $\left.r_{y_{j^{\prime}, 2}^{p}}\left(y_{j^{\prime}, 1}^{p}, y_{j^{\prime}, 3}^{p}\right)\right) \stackrel{)^{\prime}, 1}{=}(0,0,0)$. We have the following subcases.

Subcase 3.2.1: If $r_{y_{j^{\prime}, 3}^{p}}\left(y_{j^{\prime}, 2}^{p}, y_{j^{\prime}, 4}^{p}\right)=0$, then $u \in F$. Assume that $u \notin F$ by contradiction. We have $\left\{y_{j^{\prime}, 1}^{p}, y_{j^{\prime}, 2}^{p}, y_{j^{\prime}, 3}^{p}\right\} \subseteq F$. By Proposition 1, $|F| \geq 2 m_{1}+(t-1-$ $\left.m_{0}-m_{1}\right)+(t-1)+3=2 t+m_{1} \geq$ $2 t>2 t-1$, which contradicts the assumption that $|F| \leq 2 t-1$.

Subcase 3.2.2:

$\left(r_{y_{j^{\prime}, 3}^{p}}^{p}\left(y_{j^{\prime}, 2}^{p}, y_{j^{\prime}, 4}^{p}\right), r_{z_{j^{\prime}}^{p}}\left(y_{j^{\prime}, 1}^{p}, w_{j^{\prime}, 1}^{p}\right)\right.$ $\left.r_{w_{j^{\prime}, 1}^{p}}^{p^{\prime}, 3}\left(z_{j^{\prime}}^{p}, w_{j^{\prime}, 2}^{p}\right)\right)=(1,0,0)$, then $u \in F$. Assume that $u \notin F$ by contradiction. We have $\left\{y_{j^{\prime}, 1}^{p}, z_{j^{\prime}}^{p}, w_{j^{\prime}, 1}^{p}\right\} \subseteq F$. By Proposition 1, $|F| \geq 2 m_{1}+\left(t-1-m_{0}-\right.$ $\left.m_{1}\right)+(t-1)+3=2 t+m_{1} \geq 2 t>2 t-1$, 
which contradicts the assumption that $|F| \leq 2 t-1$.

Subcase

3.2.3:

If

$\left(r_{y_{j^{\prime}, 3}^{p}}^{p}\left(y_{j^{\prime}, 2}^{p}, y_{j^{\prime}, 4}^{p}\right), r_{z_{j^{\prime}}^{p}}\left(y_{j^{\prime}, 1}^{p}, w_{j^{\prime}, 1}^{p}\right), r_{w_{j^{\prime}, 1}^{p}}\right.$ $\left.\left(z_{j^{\prime}}^{p^{\prime}, w_{j^{\prime}, 2}^{p}}\right)\right)=(1,0,1)$, then $u \notin \stackrel{j^{\prime}}{F}$. Assume that $u \in F$ by contradiction. Because $r_{y_{j^{\prime}, 3}^{p}}\left(y_{j^{\prime}, 2}^{p}, y_{j^{\prime}, 4}^{p}\right)=1$, and $r_{w_{j^{\prime}, 1}^{p}}\left(z_{j^{\prime}}^{p}, w_{j^{\prime}, 2}^{p}\right)=1$, thus there is at least one faulty vertex in $\left\{y_{j^{\prime}, 2}^{p}, y_{j^{\prime}, 3}^{p}, y_{j^{\prime}, 4}^{p}\right\}$, and in $\left\{z_{j^{\prime}}^{p}, w_{j^{\prime}, 1}^{p}, w_{j^{\prime}, 2}^{p}\right\}$, respectively. By Proposition 1, $|F| \geq 2 m_{1}+\left(t-1-m_{0}-\right.$ $\left.m_{1}\right)+(t-1)+3=2 t+m_{1} \geq 2 t>2 t-1$, which contradicts the assumption that $|F| \leq 2 t-1$.

Subcase 3.2.4: If $r_{y_{j^{\prime}, 3}^{p}}\left(y_{j^{\prime}, 2}^{p}, y_{j^{\prime}, 4}^{p}\right)=1$, and $r_{z_{j^{\prime}}^{p}}\left(y_{j^{\prime}, 1}^{p}, w_{j^{\prime}, 1}^{p}\right) \stackrel{j^{\prime}, 3}{=} 1$, then $u \notin F$. Assume that $u \in F$ by contradiction. Because $r_{y_{j^{\prime}, 3}^{p}}\left(y_{j^{\prime}, 2}^{p}, y_{j^{\prime}, 4}^{p}\right)=1$, and $r_{z_{j^{\prime}}^{p}}\left(y_{j^{\prime}, 1}^{p}, w_{j^{\prime}, 1}^{p}\right)=1$, thus there is at least one faulty vertex in $\left\{y_{j^{\prime}, 2}^{p}, y_{j^{\prime}, 3}^{p}, y_{j^{\prime}, 4}^{p}\right\}$, and in $\left\{y_{j^{\prime}, 1}^{p}, w_{j^{\prime}, 1}^{p}, z_{j^{\prime}}^{p}\right\}$, respectively. By Proposition 1, $|F| \geq 2 m_{1}+\left(t-1-m_{0}-\right.$ $\left.m_{1}\right)+(t-1)+3=2 t+m_{1} \geq 2 t>2 t-1$, which contradicts the assumption that $|F| \leq 2 t-1$.

Subcase 3.3: Suppose that $m_{0}=0$. We have the following subcases.

Subcase 3.3.1: If there exists some $j^{\prime}$ such that $\left(r_{y_{j^{\prime}, 2}^{p}}\left(y_{j^{\prime}, 1}^{p}, y_{j^{\prime}, 3}^{p}\right), r_{y_{j^{\prime}, 3}^{p}}\left(y_{j^{\prime}, 2}^{p}, y_{j^{\prime}, 4}^{p}\right)\right)=$ $(0,0)$, then $u \in F$. Assume that $u \notin F$ by contradiction. We have $y_{j^{\prime}, 1}^{p} \in F$. Because $r_{y_{j^{\prime}, 2}^{p}}\left(y_{j^{\prime}, 1}^{p}, y_{j^{\prime}, 3}^{p}\right)=0$, and $r_{y_{j^{\prime}, 3}^{p}}\left(y_{j^{\prime}, 2}^{p}, y_{j^{\prime}, 4}^{p}\right)^{j^{, 2}}=0$, thus $\left\{y_{j^{\prime}, 2}^{p}, y_{j^{\prime}, 3}^{p^{\prime}, 3}\right\} \subseteq F$. By Proposition $1,|F| \geq$ $(t-1)+(t-1)+2=2 t \geq 2 t>2 t-1$, which contradicts the assumption that $|F| \leq 2 t-1$.

Subcase 3.3.2: Suppose that either $r_{y_{j, 2}^{p}}\left(y_{j, 1}^{p}, y_{j, 3}^{p}\right)=1$ or $r_{y_{j, 3}^{p}}\left(y_{j, 2}^{p}, y_{j, 4}^{p}\right)=1$ for all $1 \leq j \leq t-1$. There exists at least one faulty vertex in $\left\{y_{j, 1}^{p}, y_{j, 2}^{p}, y_{j, 3}^{p}, y_{j, 4}^{p}\right\}$. We have the following subcases.

Subcase 3.3.2.1: If there exists some $j^{\prime}$ such that $r_{w_{j^{\prime}, 1}^{p}}\left(z_{j^{\prime}}^{p}, w_{j^{\prime}, 2}^{p}\right)=1$, then $u \notin F$. Assume that $u \in F$ by contradiction. Because $r_{w_{j^{\prime} 1}^{p}}\left(z_{j^{\prime}}^{p}, w_{j^{\prime}, 2}^{p}\right)=$ 1 , thus there exists at least one faulty vertex in $\left\{z_{j^{\prime}}^{p}, w_{j^{\prime}, 1}^{p}, w_{j^{\prime}, 2}^{p}\right\}$. By Proposition $1,|F| \geq(t-1)+(t-1)+2=$ $2 t \geq 2 t>2 t-1$, which contradicts the assumption that $|F| \leq 2 t-1$.

Subcase 3.3.2.2: If $r_{w_{j, 1}^{p}}\left(z_{j}^{p}, w_{j, 2}^{p}\right)=0$ for all $1 \leq j \leq t-1$, and there exists some $j^{\prime}$ such that $r_{z_{j^{\prime}}^{p}}\left(y_{j^{\prime}, 1}^{p}, w_{j^{\prime}, 1}^{p}\right)=0$, then $u \in F$. Assume that $u \notin F$ by contradiction. We have $y_{j^{\prime}, 1}^{p} \in F$. Because $\left(r_{w_{j, 1}^{p}}\left(z_{j}^{p}, w_{j, 2}^{p}\right), r_{z_{j^{\prime}}^{p}}\left(y_{j^{\prime}, 1}^{p}, w_{j^{\prime}, 1}^{p}\right)\right)=$ $(0,0)$, there exist at least two faulty vertices in $\left\{z_{j^{\prime}}^{p}, w_{j^{\prime}, 1}^{p}, w_{j^{\prime}, 2}^{p}\right\}$.

By Proposition $1,|F| \geq(t-1)+(t-$ 1) $+2=2 t \geq 2 t>2 t-1$, which contradicts the assumption that $|F| \leq$ $2 t-1$.

Subcase

If

$\left(r_{w_{j, 1}^{p}}\left(z_{j}^{p}, w_{j, 2}^{p}\right), r_{z_{j}^{p}}\left(y_{j, 1}^{p}, w_{j, 1}^{p}\right)\right)=$ $(0,1)$ for all $1 \leq j \leq t-1$, then $u \notin F$. Assume that $u \in F$ by contradiction. Because $N_{G}\left(x^{p}\right) \nsubseteq F$, there exists some $j^{\prime}$ such that $y_{j^{\prime}, 1}^{p} \notin F$. Because $\left(r_{w_{j^{\prime}, 1}^{p}}\left(z_{j^{\prime}}^{p}, w_{j^{\prime}, 2}^{p}\right), r_{z_{j^{\prime}}^{p}}\left(y_{j^{\prime}, 1}^{p}, w_{j^{\prime}, 1}^{p}\right)\right)=$ $(0,1)$, there is at least one faulty vertex in $\left\{w_{j^{\prime}, 1}^{p}, z_{j^{\prime}}^{p}\right\}$. By Proposition 1, $|F| \geq(t-1)+(t-1)+2=2 t \geq$ $2 t>2 t-1$, which contradicts the assumption that $|F| \leq 2 t-1$.

Thus the algorithm CFLDA1 $\left(B W B_{G}(u ; t), S\right)$ is correct.

Case 4) Suppose that $|S|=0$. We prove the correctness of the algorithm CFLDA0 $\left(B W B_{G}(u ; t), S\right)$ as follows. For every $1 \leq i \leq t$ and $1 \leq j \leq t-1$, let

$$
\begin{gathered}
n_{0}^{i}=\mid\left\{j \mid\left(r_{y_{j, 1}^{i}}\left(x^{i}, z_{j}^{i}\right), r_{y_{j, 1}^{i}}\left(x^{i}, y_{j, 2}^{i}\right), r_{y_{j, 2}^{i}}\left(y_{j, 1}^{i}, y_{j, 3}^{i}\right),\right.\right. \\
\left.\left.r_{y_{j, 3}^{i}}\left(y_{j, 2}^{i}, y_{j, 4}^{i}\right)\right)=(0,0,0,0)\right\} \mid, \\
n_{1}^{i}=\mid\left\{j \mid\left(r_{y_{j, 1}^{i}}\left(x^{i}, z_{j}^{i}\right), r_{y_{j, 1}^{i}}\left(x^{i}, y_{j, 2}^{i}\right), r_{y_{j, 2}^{i}}\left(y_{j, 1}^{i}, y_{j, 3}^{i}\right),\right.\right. \\
\left.\left.r_{y_{j, 3}^{i}}\left(y_{j, 2}^{i}, y_{j, 4}^{i}\right)\right)=(0,0,0,1)\right\} \mid, \text { and }
\end{gathered}
$$

Because $|S|=0$, thus $n_{2}^{i}>n_{0}^{i}+n_{1}^{i}$. First, we claim that, if $n_{2}^{i} \geq n_{0}^{i}+n_{1}^{i}+2$ for every $1 \leq i \leq t$, then $u \in F$. Assume that $u \notin F$ by contradiction. Let $S^{\prime}=\left\{x^{i} \mid x^{i} \notin F\right.$, and $\mathbf{L D A}\left(W B_{G}\left(x^{i} ; t-1\right)\right)=$ 1\}. By Lemma $1,\left|S^{\prime}\right| \leq 1$. Suppose that $\left|S^{\prime}\right|=1$, and $x^{j} \in S$. Then by Proposition 1 , we have $|F| \geq$ $2 n_{2}^{j}+\left(t-1-n_{0}^{j}-n_{1}^{j}-n_{2}^{j}\right)+(t-1)=2 t-2+$ $n_{2}^{j}-n_{1}^{j}-n_{0}^{j} \geq 2 t>2 t-1$, which contradicts the assumption that $|F| \leq 2 t-1$. Suppose that $\left|S^{\prime}\right|=0$. Because $N_{G}(u) \subseteq F$, we have $u \in F$.

Now we claim that there is at most one index $i$ for $1 \leq i \leq t$ such that $n_{2}^{i}=n_{0}^{i}+n_{1}^{i}+1$. By contradiction, assume that $n_{2}^{i_{1}}=n_{0}^{i_{1}}+n_{1}^{i_{1}}+1$, and $n_{2}^{i_{2}}=n_{0}^{i_{2}}+n_{1}^{i_{2}}+1$ for some $1 \leq i_{1} \leq t$, and $1 \leq i_{2} \leq t$. We have two cases. First, the two vertices $x^{\bar{i}_{1}}$ and $x^{i_{2}}$ are faulty. Suppose that $\left\{x^{i_{1}}, x^{i_{2}}\right\} \subseteq F$. By Proposition 1, we have $|F| \geq$ $2\left(n_{0}^{i_{1}}+n_{1}^{i_{1}}\right)+\left(t-1-n_{0}^{i_{1}}-n_{1}^{i_{1}}-n_{2}^{i_{1}}\right)+2\left(n_{0}^{i_{2}}+\right.$ $\left.n_{1}^{i_{2}}\right)+\left(t-1-n_{0}^{i_{2}}-n_{1}^{i_{2}}-n_{2}^{i_{2}}\right)+(t-2)+2=\left(n_{0}^{i_{1}}+\right.$ $\left.n_{1}^{i_{1}}-n_{2}^{i_{1}}\right)+\left(n_{0}^{i_{2}}+n_{1}^{i_{2}}-n_{2}^{i_{2}}\right)+(3 t-2)=3 t-4>$ 
$2 t-1$ if $t \geq 5$, which contradicts the assumption that $|F| \leq 2 t-1$. Second, one of $x^{i_{1}}$ and $x^{i_{2}}$ is faulty. Without loss of generality, suppose that $x^{i_{1}} \in F$ and $x^{i_{2}} \in S^{\prime}$. By Proposition 1, we have $|F| \geq 2\left(n_{0}^{i_{1}}+n_{1}^{i_{1}}\right)+\left(t-1-n_{0}^{i_{1}}-n_{1}^{i_{1}}-n_{2}^{i_{1}}\right)+2 n_{2}^{i_{2}}+$ $\left(t-1-n_{0}^{i_{2}}-n_{1}^{i_{2}}-n_{2}^{i_{2}}\right)+(t-2)+1=\left(n_{0}^{i_{1}}+\right.$ $\left.n_{1}^{i_{1}}-n_{2}^{i_{1}}\right)+\left(n_{2}^{i_{2}}-n_{1}^{i_{2}}-n_{0}^{i_{2}}\right)+(3 t-3)=3 t-3>$ $2 t-1$ if $t \geq 5$, which contradicts the assumption that $|F| \leq 2 t-1$. Hence let $i^{\prime}$ be the only index such that $n_{2}^{i^{\prime}}=n_{0}^{i^{\prime}}+n_{1}^{i^{\prime}}+1$, where $1 \leq i^{\prime} \leq t$. We have the following subcases.

Subcase 4.1: If $n_{0}^{i^{\prime}}=0$ and $n_{1}^{i^{\prime}} \neq 0$, then $u \in$ $F$. We claim that $x^{i^{\prime}} \in F$. Assume that $x^{i^{\prime}} \notin F$ by contradiction. Because $n_{1}^{i^{\prime}} \neq 0$, there exists some $l$ such that at least one of $\left\{y_{l, 2}^{i^{\prime}}, y_{l, 3}^{i^{\prime}}, y_{l, 4}^{i^{\prime}}\right\}$ is in fault. By Proposition 1, we have $|F| \geq$ $2 n_{2}^{i^{\prime}}+\left(t-1-n_{0}^{i^{\prime}}-n_{1}^{i^{\prime}}-n_{2}^{i^{\prime}}\right)+(t-1)+1=\left(n_{2}^{i^{\prime}}-\right.$ $\left.n_{0}^{i^{\prime}}-n_{1}^{i^{\prime}}\right)+(2 t-1)=2 t>2 t-1$, which contradicts the assumption that $|F| \leq 2 t-1$. Thus $x^{i^{\prime}} \in F$. Because $N_{G}(u) \subseteq F$, we have $u \in F$.

Subcase 4.2: Suppose that $n_{0}^{i^{\prime}}=0$, and $n_{1}^{i^{\prime}}=0$. Hence, we have $n_{2}^{i^{\prime}}=1$. Let $j^{\prime}$ be the index such that $\left(r_{y_{j^{\prime}, 1}^{i^{\prime}}}\left(x^{i^{\prime}}, z_{j^{\prime}}^{i^{\prime}}\right), r_{y_{j^{\prime}, 1}^{i^{\prime}}}\left(x^{i^{\prime}}, y_{j^{\prime}, 2}^{i^{\prime}}\right), r_{y_{j^{\prime}, 2}^{i^{\prime}}}\left(y_{j^{\prime}, 1}^{i^{\prime}},=\right.\right.$ $\left.y_{j^{\prime}, 3}^{i^{\prime}}\right)(1,1,0)$. We prove that if $r_{y_{j^{\prime}, 3}^{i^{\prime}}}\left(y_{j^{\prime}, 2}^{i^{\prime}}, y_{j^{\prime}, 4}^{i^{\prime}}\right)=1$, then $u \notin F$. Assume that $u \in F$ by contradiction. Because $r_{z_{j^{\prime}}^{i^{\prime}}}\left(x^{i^{\prime}}, y_{j^{\prime}, 1}^{i^{\prime}}\right)=1$, and $r_{y_{j^{\prime}, 3}^{i^{\prime}}}\left(y_{j^{\prime}, 2}^{i^{\prime}}, y_{j^{\prime}, 4}^{i^{\prime}}\right)=1$, there is least one faulty vertex in $\left\{x^{i^{\prime}}, y_{j^{\prime}, 1}^{i^{\prime}}, z_{j^{\prime}}^{i^{\prime}}\right\}$, and in $\left\{y_{j^{\prime}, 2}^{i^{\prime}}, y_{j^{\prime}, 3}^{i^{\prime}}, y_{j^{\prime}, 4}^{i^{\prime}}\right\}$, respectively. By Proposition 1, we have $|F| \geq\left(t-1-n_{0}^{i^{\prime}}-n_{1}^{i^{\prime}}-n_{2}^{i^{\prime}}\right)+(t-1)+3=$ $2 t>2 t-1$, which contradicts the assumption that $|F| \leq 2 t-1$. Now we prove that if $r_{y_{j^{\prime}, 3}^{i^{\prime}}}\left(y_{j^{\prime}, 2}^{i^{\prime}}, y_{j^{\prime}, 4}^{i^{\prime}}\right)=0$, then $u \in F$. We claim that $y_{j^{\prime}, 1}^{i^{\prime}} \notin F$. Assume that $y_{j^{\prime}, 1}^{i^{\prime}} \in F$ by contradiction. Because $r_{y_{j^{\prime}, 2}^{i^{\prime}}}\left(y_{j^{\prime}, 1}^{i^{\prime}}, y_{j^{\prime}, 3}^{i^{\prime}}\right)=0$, and $r_{y_{j^{\prime}, 3}^{i^{\prime}}}\left(y_{j^{\prime}, 2}^{i^{\prime}}, y_{j^{\prime}, 4}^{i^{\prime}}\right) \stackrel{j^{\prime}, 2}{=} 0$, we have $\left\{y_{j^{\prime}, 2}^{i^{\prime}}, y_{j^{\prime}, 3}^{i^{\prime}, 3}\right\} \subseteq F$. By Proposition 1, we have $|F| \geq\left(t-1-n_{0}^{i^{\prime}}-n_{1}^{i^{\prime}}-n_{2}^{i^{\prime}}\right)+(t-1)+3=$ $2 t>2 t-1$, which contradicts the assumption that $|F| \leq 2 t-1$. Thus, $y_{j^{\prime}, 1}^{i^{\prime}} \notin F$. Then we claim that $x^{i^{\prime}} \in F$. Assume that $x^{i^{\prime}} \notin F$ by contradiction. Because $r_{y_{j^{\prime}, 1}^{i^{\prime}}}\left(x^{i^{\prime}}, z_{j^{\prime}}^{i^{\prime}}\right)=1, r_{y_{j^{\prime}, 1}^{i^{\prime}}}\left(x^{i^{\prime}}, y_{j^{\prime}, 2}^{i^{\prime}}\right)=1$, and $r_{y_{j^{\prime}, 3}^{i^{\prime}}}\left(y_{j^{\prime}, 2}^{i^{\prime}}, y_{j^{\prime}, 4}^{i^{\prime}}\right)=0$, we have $\left\{y_{j^{\prime}, 2}^{i^{\prime}}, y_{j^{\prime}, 3}^{i^{\prime}}, z_{j^{\prime}}^{i^{\prime}}\right\} \subseteq F$. By Proposition 1, we have $|F| \geq\left(t-1-n_{0}^{i^{\prime}}-n_{1}^{i^{\prime}}-n_{2}^{i^{\prime}}\right)+(t-1)+3=$ $2 t>2 t-1$, which contradicts the assumption that $|F| \leq 2 t-1$. Thus, $x^{i^{\prime}} \in F$. Because $N_{G}(u) \subseteq F$, we have $u \in F$.

Subcase 4.3: Suppose that $n_{0}^{i^{\prime}} \neq 0$. We claim that $x^{i^{\prime}} \notin F$. Assume that $x^{i^{\prime}} \in \quad F$ by contradiction. Because $\left(r_{y_{l^{\prime}, 1}^{i^{\prime}}}\left(x^{i^{\prime}}, z_{l^{\prime}}^{i^{\prime}}\right), r_{y_{l^{\prime}, 1}^{i^{\prime}}}\left(x^{i^{\prime}}, y_{l^{\prime}, 2}^{i^{\prime}}\right), r_{y_{l^{\prime}, 2}^{i^{\prime}}}\right.$ $\left.\left(y_{l^{\prime}, 1}^{i^{\prime}}, y_{l^{\prime}, 3}^{i^{\prime}}\right), r_{y_{l^{\prime}, 3}^{i^{\prime}}}\left(y_{l^{\prime}, 2}^{i^{\prime}}, y_{l^{\prime}, 4}^{i^{\prime}}\right)\right)=(0,0,0,0)$ for some $1 \stackrel{\leq}{\leq} l^{\prime} \leq t-1$, we have $\left\{u, y_{l^{\prime}, 1}^{i^{\prime}}, y_{l^{\prime}, 2}^{i^{\prime}}, y_{l^{\prime}, 3}^{i^{\prime}}\right\} \subseteq F$. By Proposition 1, we have $|F| \geq 2\left(n_{0}^{i^{\prime}}+n_{1}^{i^{\prime}}\right)+\left(t-1-n_{0}^{i^{\prime}}-n_{1}^{i^{\prime}}-\right.$ $\left.n_{2}^{i^{\prime}}\right)+(t-1)+4=\left(n_{0}^{i^{\prime}}+n_{1}^{i^{\prime}}-n_{2}^{i^{\prime}}\right)+(2 t+2)=$ $2 t+1>2 t-1$, which contradicts the assumption that $|F| \leq 2 t-1$. Thus, $x^{i^{\prime}} \notin F$. Hence, if there exists some $j^{\prime \prime}$ such that $r_{x^{i^{\prime}}}\left(u, y_{j^{\prime \prime}, 1}^{i^{\prime}}\right)=0$ for $1 \leq j^{\prime \prime} \leq t-1$, then $u \notin F$. Now we prove that if $r_{x^{i^{\prime}}}\left(u, y_{j, 1}^{i^{\prime}}\right)=1$ for all $1 \leq j \leq t-1$, then $u \in F$. Assume that $u \notin F$ by contradiction. We have $y_{j, 1}^{i^{\prime}} \in F$ for all $1 \leq j \leq t-1$. Because $n_{0}^{i^{\prime}} \neq 0$, there exists some $l^{\prime \prime}$ such that $r_{y_{l^{\prime \prime}, 3}^{i^{\prime}}}\left(y_{l^{\prime \prime}, 2}^{i^{\prime}}, y_{l^{\prime \prime}, 4}^{i^{\prime}}\right)=0$. Thus, we have $y_{l^{\prime \prime}, 3}^{i^{\prime}} \in F$. By Proposition 1, we have $|F| \geq 2\left(n_{0}^{i^{\prime}}+n_{1}^{i^{\prime}}\right)+n_{2}^{i^{\prime}}+\left(t-1-n_{0}^{i^{\prime}}-n_{1}^{i^{\prime}}-\right.$ $\left.n_{2}^{i^{\prime}}\right)+(t-1)+1=\left(n_{0}^{i^{\prime}}+n_{1}^{i^{\prime}}\right)+(2 t-1)>2 t-1$, which contradicts the assumption that $|F| \leq 2 t-1$. Thus, $u \in F$.

Therefore, the algorithm CFLDA0 $\left(B W B_{G}(u ; t), S\right)$ is correct.

\section{REFERENCES}

[1] S. B. Akers and B. Krishnameurthy, "Group-theoretic model for symmetric interconnection networks," IEEE Trans. Comput., vol. 38, no. 4, pp. 555-566, Apr. 1989.

[2] N. Bagherzadeh, M. Dowd, and N. Nassif, "Embedding an arbitrary tree into the star graph," IEEE Trans. Comput., vol. 45, no. 4, pp. 475-481, Apr. 1996.

[3] T. A. Bartic, J. Y. Mignolet, V. Nollet, T. Marescaux, D. Verkest, S. Vernalde, and R. Lauwereins, "Topology adaptive networkon-chip design and implementation," IEE Proc. Comput. Digital Techniq., vol. 152 , no. 4, July 2005.

[4] P. Berthome, A. Ferreira, and S. Perennes, "Optimal information dissemination in star and pancake networks," IEEE Trans. Parallel Distrib. Syst., vol. 7, no. 12, pp. 1292-1300, Dec. 1996.

[5] C. P. Chang, P. L. Lai, J. J. M. Tan, and L. H. Hsu, "Diagnosability of $t$-connected networks and product networks under the comparison diagnosis model," IEEE Trans. Comput., vol. 53, no. 12, pp. 1582-1590, Dec. 2004.

[6] G. Y. Chang and G. H. Chen, “ $(t, k)$-diagnosability of multiprocessor systems with applications to grids and tori," SIAM J. Comput., vol. 37, pp. 1280-1298, 2007.

[7] C. F. Chiang and J. J. M. Tan, "Using node diagnosability to determine $t$-diagnosability under the comparison diagnosis model," IEEE Trans. Comput., vol. 58, no. 1, pp. 251-259, Jan. 2009.

[8] A. Das, K. Thulasiraman, and V. K. Agarwal, "Diagnosis of $t /(t+1)$-diagnosable systems," SIAM J. Comput., vol. 23, pp. 895-905, 1994.

[9] J. Fan, "Diagnosability of crossed cubes under the comparison diagnosis model," IEEE Trans. Parallel Distrib. Syst., vol. 13, no. 7, pp. 687-692, 2002.

[10] P. Fragopoulou and S. G. Akl, "A parallel algorithm for computing Fourier transforms on the star graph," IEEE Trans. Parallel Distrib. Syst., vol. 5, no. 5, pp. 525-531, 1994.

[11] P. Fragopoulou and S. G. Akl, "Optimal communication algorithms on star graphs using spanning tree constructions," J. Parallel Distrib. Comput., vol. 24, pp. 55-71, 1995.

[12] W. S. Hong and S. Y. Hsieh, "Strong diagnosability and conditional diagnosability of augmented cubes under the comparison diagnosis model," IEEE Trans. Rel., vol. 61, no. 1, pp. 140-148, Mar. 2011.

[13] S. Y. Hsieh and Y. S. Chen, "Strongly diagnosable product networks under the comparison diagnosis model," IEEE Trans. Comput., vol. 57 , no. 6, pp. 721-732, Jun. 2008. 
[14] S. Y. Hsieh and Y. S. Chen, "Strongly diagnosable systems under the comparison diagnosis model," IEEE Trans. Comput., vol. 57, no. 12, pp. $1720-1725$, Dec. 2008.

[15] S. Y. Hsieh and T. Y. Chuang, "The strong diagnosability of regular networks and product networks under the PMC model," IEEE Trans. Parallel Distrib. Syst., vol. 20, no. 3, pp. 367-378, Mar. 2009.

[16] G. H. Hsu and J. J. M. Tan, "A local diagnosability measure for multiprocessor systems," IEEE Trans. Parallel Distributed Syst., vol. 18, no. 5, pp. 598-607, May 2007.

[17] L. H. Hsu and C. K. Lin, Graph Theory and Interconnection Networks. Boca Raton, FL, USA: CRC Press, 2008.

[18] Y. Ishida, N. Adachi, and H. Tokumaru, "Diagnosability and distinguishability analysis and its applications," IEEE Trans. Rel., vol. 36, no. 5, pp. 531-538, Dec. 1987.

[19] J. S. Jwo, S. Lakshmivarahan, and S. K. Dhall, "Embedding of cycles and grids in star graphs," J. Circuits, Syst., Comput., vol. 1, pp. 43-74, 1991.

[20] P. L. Lai, J. J. M. Tan, C. P. Chang, and L. H. Hsu, "Conditional diagnosability measures for large multiprocessor systems," IEEE Trans. Comput., vol. 54, no. 2, pp. 165-175, Feb. 2005.

[21] C. W. Lee and S. Y. Hsieh, "Diagnosability of two-matching composition networks under the MM* model," IEEE Trans. Dependable Secure Comp., vol. 8, no. 2, pp. 246-255, 2011.

[22] C. W. Lee and S. Y. Hsieh, "Determining the diagnosability of $(1,2)$ matching composition networks and its applications," IEEE Trans. Dependable Secure Comput., vol. 8, no. 3, pp. 353-362, 2011.

[23] C. K. Lin, T. L. Kung, and J. J. M. Tan, "Conditional-fault diagnosability of multiprocessor systems with an efficient local diagnosis algorithm under the PMC model," IEEE Trans. Parallel Distrib. Syst., vol. 22, no. 10, pp. 1669-1680, Oct. 2011.

[24] J. Maeng and M. Malek, "A comparison connection assignment for self-diagnosis of multiprocessors systems," in Proc. 11th Int. Symp. Fault-Tolerant Comput. (FTCS '81), 1981, pp. 173-175.

[25] M. Malek, "A comparison connection assignment for diagnosis of multiprocessors systems," in Proc. 7th Int. Symp. Comput. Architecture (ISCA '80), 1980, pp. 31-36.

[26] V. E. Mendia and D. Sarkar, "Optimal broadcasting on the star graph," IEEE Trans. Parallel Distrib. Syst., vol. 3, no. 4, pp. 389-396, Apr. 1992.

[27] Z. Miller, D. Pritikin, and I. H. Sudborough, "Near embeddings of hyperucbes into Cayley graphs on the symmetric group," IEEE Trans. Comput., vol. 43, no. 1, pp. 13-22, Jan. 1994.

[28] P. P. Pande, C. Grecu, M. Jones, A. Ivonov, and R. Saleh, "Performance evaluation and design trade-offs for network-on-chip interconnect architectures," IEEE Trans. Comput., vol. 54, no. 8, pp. 1025-1040, Aug. 2005.

[29] F. P. Preparata, G. Metze, and R. T. Chien, "On the connection assignment problem of diagnosis systems," IEEE Trans. Electron. Comput., vol. 16 , no. 12 , pp. 848-854, Dec. 1967.
[30] A. Sengupta and A. Dahbura, "On self-diagnosable multiprocessor system: Diagnosis by the comparison approach," IEEE Trans. Comput., vol. 41, no. 11, pp. 1386-1396, Nov. 1992

[31] S. A. Wong, "Hamiltonian cycles and paths in butterfly graphs," Networks, vol. 26, pp. 145-150, 1995.

[32] J. Zhao, F. J. Meyer, N. Park, and F. Lombardi, "Sequential diagnosis of processor array systems," IEEE Trans. Rel., vol. 53, no. 4, pp. 487-498, Dec. 2004

[33] J. Zheng, S. Latifi, E. Regentova, K. Luo, and X. Wu, "Diagnosability of star graphs under the comparison diagnosis model," Inf. Process. Lett., vol. 93, pp. 29-36, 2005.

Cheng-Kuan Lin received the BS degree in applied mathematics from Chinese Culture University, Taiwan, Republic of China, in 2000; the MS degree in mathematics from the National Central University in 2002; and the PhD degree in computer science from the National Chiao Tung University in 2011 . He is now a postdoctoral fellow in the Institute of Information Science, Academia Sinica His research interests include interconnection network, algorithm, graph theory, wireless network, and wireless sensor network.

Yuan-Hsiang Teng received a B.S. degree, and an M.S. degree in computer information science from the National Chiao Tung University, Taiwan, in 2002 , and 2004, respectively. He received a Ph.D. degree in computer science from the National Chiao Tung University in 2008. Currently, he is an assistant professor in the Department of Computer Science and Information Engineering, Hungkuang University, Taiwan, Republic of China. His research interests include graph theory, interconnection networks, and algorithms.

Jimmy J. M. Tan received the BS and MS degrees in mathematics from the National Taiwan University in 1970 and 1973, respectively, and the $\mathrm{PhD}$ degree from Carleton University, Ottawa, in 1981. He has been on the faculty of the Department of Computer Science, National Chiao Tung University, since 1983. His research interests include design and analysis of algorithms, combinatorial optimization, and interconnection networks.

Lih-Hsing Hsu received the BS degree in mathematics from Chung Yuan Christian University, Taiwan, Republic of China, in 1975 and the $\mathrm{PhD}$ degree in mathematics from the State University of New York at Stony Brook in 1981. He is currently a professor in the Department of Computer Science and Information Engineering, Providence University, Taiwan, Republic of China. His research Interests include interconnection networks, algorithms, graph theory, and VLSI layout. 